\title{
Correlação entre índice de esforço percebido e respostas cardiopulmonares em exercício de hidroginástica com equipamento
}

\section{Correlation between rate of perceived exertion and cardiopulmonary responses in water exercise performed with equipment}

Stephanie Santana Pinto ${ }^{1,2}$

Paula Finatto

Cristine Lima Alberton ${ }^{1,2}$

Luiz Fernando Martins Kruel ${ }^{1}$

\section{Resumo}

O objetivo deste estudo foi analisar a correlação entre o índice de esforço percebido e as variáveis cardiopulmonares durante a execução de um exercício de hidroginástica nas situações de uso de equipamento resistido Aquafins ${ }^{\oplus}$ nos membros superiores, equipamento resistido nos membros inferiores e equipamento resistido nos membros superiores e inferiores simultaneamente. Onze mulheres participaram de três sessões: uma sessão para caracterização da amostra e teste de esforço máximo, uma de familiarização e outra correspondente ao protocolo experimental. $\mathrm{O}$ protocolo experimental consistiu da execução do exercício deslize frontal combinado com a flexão e extensão horizontal de ombros, durante quatro minutos na cadência de 60 batidas por minuto, nas três situações de uso de equipamento resistido. Para correlacionar o índice de esforço percebido (IEP) com a frequência cardíaca, percentual da frequência cardíaca máxima, consumo de oxigênio, percentual do consumo de oxigênio máximo e ventilação, utilizou-se a correlação de Spearman, com $\alpha=5 \%$. Observou-se correlações significativas ( $r h o=0,60-0,88 ; p<0,05)$ entre o IEP e as variáveis cardiopulmonares na situação de uso de equipamento nos membros inferiores. Todavia, nas demais situações (com equipamento resistido nos membros inferiores e em ambos os membros) não houve correlações significativas entre esses parâmetros, com exceção do IEP e a ventilação na situação de uso de equipamento em ambos os membros ( $r h o=0,71 ; p=0,015)$. Em suma, o IEP está associado com a com a frequência cardíaca, o consumo de oxigênio e a ventilação e reflete a intensidade do exercício de hidroginástica quando realizado com equipamento resistido nos membros inferiores.

\section{Palavras-chave}

Frequência cardíaca; Consumo de oxigênio; Esforço percebido; Imersão.

\begin{abstract}
The aim of the present study was to correlate the rating of perceived exertion with cardiopulmonary variables during the performance of a water-based exercise in situations with the use of Aquafins aquatic drag equipment in upper limbs, in lower limbs and in both upper and lower limbs. Eleven women took part in three data collection sessions: sample characterization, maximal test, familiarization and experimental protocol. The experimental protocol was performed with the execution of cross country skiing combined with horizontal shoulder flexion and extension exercise during four minutes at $60 \mathrm{bpm}$ in the three situations with the use of aquatic drag equipment. Spearman correlation was used, in order to correlate the rating of perceived exertion (RPE) with heart rate, percentage of the maximal heart rate, oxygen uptake, percentage of the maximal oxygen uptake and ventilation, with $\alpha=5 \%$. The results showed significant correlations between $R P E$ and cardiopulmonary variables in the situation with the use of equipment in lower limbs (rho=0.60-0.88; $p<0.05)$. However, in the others situations (with aquatic drag equipment in lower limbs and in both upper and lower limbs) there was no relationship between these parameters, except the correlation between $R P E$ and ventilation in the situation with the use of equipment in both upper and lower limbs (rho=0.71; $p=0.015)$. In conclusion, the RPE is associated with the heart rate, oxygen uptake and ventilation and corresponds to the intensity of the water-based exercise performed with aquatic drag equipment in lower limbs.
\end{abstract}

\section{Keywords}

Heart rate; Oxygen uptake; Perceived exertion; Immersion.
Rev Bras Ativ Fis Saúde p. 475-482

DOI:

http://dx.doi.org/10.12820/rbafs.v.18n4p475

1 Escola de Educação Física da Universidade Federal do Rio Grande do Sul (ESEF-UFRGS) 2 Escola Superior de Educação Física da Universidade Federal de Pelotas (ESEF/UFPel) 


\section{INTRODUÇÃO}

As atividades aquáticas têm se tornado cada vez mais populares por sua prescrição ser viável a diferentes grupos de pessoas ${ }^{1-3}$. Um dos fatores pelo qual os exercícios aquáticos têm sido amplamente indicados, está relacionado a possibilidade de exercitar-se com um comportamento reduzido de frequência cardíaca e pressão arterial ${ }^{4-6}$. Além disso, sabe-se que o exercício no meio aquático apresenta uma menor força de impacto quando comparado ao mesmo executado em meio terrestre ${ }^{7}$. Dessa forma, os exercícios aquáticos são terapeuticamente benéficos, sendo favoráveis àqueles que possuem alguma restrição à atividades na terra ${ }^{8}$. No meio aquático a principal forma de modificar a sobrecarga mecânica do exercício de hidroginástica é através do aumento da resistência ao avanço ${ }^{9}$. Essa força de resistência pode ser alterada em um programa de exercícios através da utilização do corpo de diferentes formas na água ${ }^{10}$, pela modificação da velocidade de execução de movimentos ${ }^{11}$ ou pela execução de exercícios com diferentes equipamentos apropriados ao meio aquático ${ }^{12}$.

A intensidade do esforço no meio aquático pode ser controlada através de diversos indicadores. Entre eles, destacam-se a frequência cardíaca (FC) e o índice de esforço percebido (IEP), os quais vem sendo utilizados na prescrição de programas de hidroginástica ${ }^{1,3,13-15}$. A percepção de esforço pode ser definida como a intensidade subjetiva de esforço, tensão, desconforto e/ou fadiga que é sentida ou experimentada durante o exercício aeróbio e de força ${ }^{16}$. A prescrição através do IEP pode ser priorizada devido ao seu baixo custo, fácil utilização e adaptação a atividades dentro da água ${ }^{17}$. Além disso, o seu uso é uma opção bastante abrangente para a prescrição de exercícios para populações especiais, os quais muitas vezes fazem uso de medicamentos que interferem na resposta da FC, não se enquadrando nos critérios para a prescrição de exercícios através desse parâmetro ${ }^{18}$.

Estudos com atividades aquáticas, tais como caminhada em piscina rasa, corrida em piscina funda e exercícios de hidroginástica, têm observado correlações fortes e significativas entre o IEP e parâmetros cardiopulmonares ${ }^{19-22}$. Entretanto, nenhum estudo na literatura pesquisada investigou a associação do IEP com variáveis cardiopulmonares durante a realização de exercícios aquáticos com equipamentos apropriados a esse ambiente. Sabe-se que os equipamentos são muito utilizados em aulas de hidroginástica com intuito de diversificar e intensificar as mesmas. Dentro desse contexto, estudos verificaram a influência da utilização de tais materiais nos parâmetros fisiológicos durante a execução de exercícios específicos de hidroginástica ${ }^{13,23-25} \mathrm{e}$ observaram incrementos significativos nas respostas cardiopulmonares e no IEP da situação sem uso de equipamentos para a situação de utilização de diferentes implementos. Entretanto, nessas pesquisas, os resultados das variáveis cardiopulmonares não foram correlacionados com o IEP. Logo, seria importante estudar a relação do IEP com as variáveis cardiopulmonares juntamente com o uso de equipamentos resistidos, visto que tal relação pode vir a ser alterada com a utilização de equipamentos, dependendo do seu posicionamento nos membros superiores e inferiores e do grupo muscular prioritariamente envolvido no exercício. Sendo assim, o objetivo do presente estudo foi correlacionar o IEP com as variáveis cardiopulmonares, em situações específicas de uso de equipamento resistido na execução de um exercício de hidroginástica.

\section{MÉTODOS}

A amostra foi composta por 11 mulheres jovens ambientadas ao meio aquáti- 
co (idade: 22,0 $\pm 2,0$ anos; massa corporal: $58,3 \pm 6,5 \mathrm{~kg}$; estatura: 163,4 $\pm 7,0 \mathrm{~cm}$ ). Todas participantes eram alunas da Escola de Educação Física da Universidade Federal do Rio Grande do Sul (UFRGS) e foram selecionadas por voluntariedade através de convite verbal. Os critérios de inclusão da amostra foram os seguintes: idade entre 18 e 26 anos, estatura entre 158 e $178 \mathrm{~cm}$, ser ambientada ao meio aquático e praticar aulas de hidroginástica, no mínimo, há três meses. Como critérios de exclusão, ser fumante e apresentar problemas cardiovasculares e musculoesqueléticos, assim como fazer uso de medicação (com exceção de contraceptivo). Anteriormente ao início das sessões de avaliação, as voluntárias assinaram um termo de consentimento livre esclarecido, no qual constavam todas as informações pertinentes ao estudo. Esta pesquisa foi aprovada pelo Comitê de Ética local e conduzido de acordo com a resolução 196/96 do Conselho Nacional da Saúde.

O procedimento experimental foi constituído por três sessões. Na primeira sessão de avaliação foram obtidas as medidas de massa corporal e estatura em uma balança de alavanca, com estadiômetro acoplado (Filizola, São Paulo, Brasil, precisão de 100 g e $1 \mathrm{~mm}$, respectivamente). A seguir, foi realizado um teste de esforço máximo em esteira terrestre para determinação do consumo de oxigênio $\left(\mathrm{VO}_{2 \max }\right)$ e da frequência cardíaca máximos $\left(\mathrm{FC}_{\text {máx }}\right)$. Para tanto, os sujeitos realizaram um protocolo com uma velocidade inicial de $5 \mathrm{~km} \cdot \mathrm{h}^{-1} \mathrm{e}$ incrementos de $1 \mathrm{~km} \cdot \mathrm{h}^{-1} \mathrm{a}$ cada minuto, com inclinação fixa de $1 \%$. O teste foi considerado válido quando um dos seguintes critérios fosse alcançado: $\mathrm{FC}_{\text {máx }}$ maior do que a estimada por idade, taxa de troca respiratória maior que 1,10 e frequência respiratória maior que 35 respirações por minuto ${ }^{26}$.

$\mathrm{Na}$ segunda sessão, foi realizada uma familiarização com o exercício e o equipamento utilizado no protocolo experimental. Além disso, os sujeitos foram familiarizados a Escala de Borg para Dor e Esforço Percebido (15 pontos, 6-20) ${ }^{27}$. Inicialmente, foram dadas instruções sobre o uso da referida escala, de acordo com as recomendações de Borg ${ }^{27}$. Na sequência, os sujeitos praticaram o exercício de hidroginástica com o uso de equipamento em diferentes níveis de esforço, variando progressivamente dos IEPs 6 a 20. É importante salientar que foi esclarecido aos indivíduos que o IEP 6 é considerado a ausência de qualquer esforço, enquanto o IEP 19 é considerado um esforço máximo. O IEP 20 é considerado uma percepção de esforço sentida apenas em casos extremos (luta ou fuga ${ }^{27}$.

O protocolo experimental foi realizado $48 \mathrm{~h}$ após a familiarização. Nessa sessão, os indivíduos realizaram o exercício deslize frontal combinado com a flexão e extensão horizontal de ombros. A fase 1 do movimento de membros superiores correspondeu a flexão horizontal de ombros e do movimento de membros inferiores, a flexão de quadril direito através de deslize. A fase 2 do movimento de membros superiores correspondeu a extensão horizontal de ombros e do movimento de membros inferiores, a extensão de quadril direito através de deslize. $\mathrm{O}$ movimento de membros inferiores foi realizado sempre de forma alternada. Esse exercício foi realizado na cadência de 60 batidas por minuto (bpm), que foi controlada com o auxílio de um metrônomo (modelo MATRIX MR-500, QUARTZ, Tóquio, Japão). Dessa forma, cada movimento completo (fases 1 e 2) foi executado em duas batidas no ritmo proposto $(60 \mathrm{bpm})$. Logo, cada voluntária realizava 30 movimentos completos por minuto. Esse exercício foi realizado durante quatro minutos nas seguintes situações de uso do equipamento resistido Aquafins ${ }^{\circledR}$ (The Hygenic Corporation, Ohio, USA): equipamento resistido nos membros superiores (MS), equipamento resistido nos membros inferiores (MI) e equipamento resistido nos membros superiores e inferiores simultaneamente (MS/MI) (figura 1). As situações foram randomizadas e respeitaram intervalos de $20 \mathrm{~min}$ em repouso fora da 
água, de acordo com estudo prévio ${ }^{12}$. O protocolo experimental foi realizado no período da tarde, em uma piscina com profundidade entre 1,10 e 1,40 m, permitindo que a profundidade de imersão ficasse entre o processo xifoide e ombros. A temperatura da água foi mantida em $32^{\circ} \mathrm{C}$. As voluntárias deveriam alimentar-se no período de 3 a $4 \mathrm{~h}$ antes do início da sessão de testes, sem a ingestão de estimulantes e evitar a prática de atividades físicas intensas durante as últimas $24 \mathrm{~h}^{28}$.

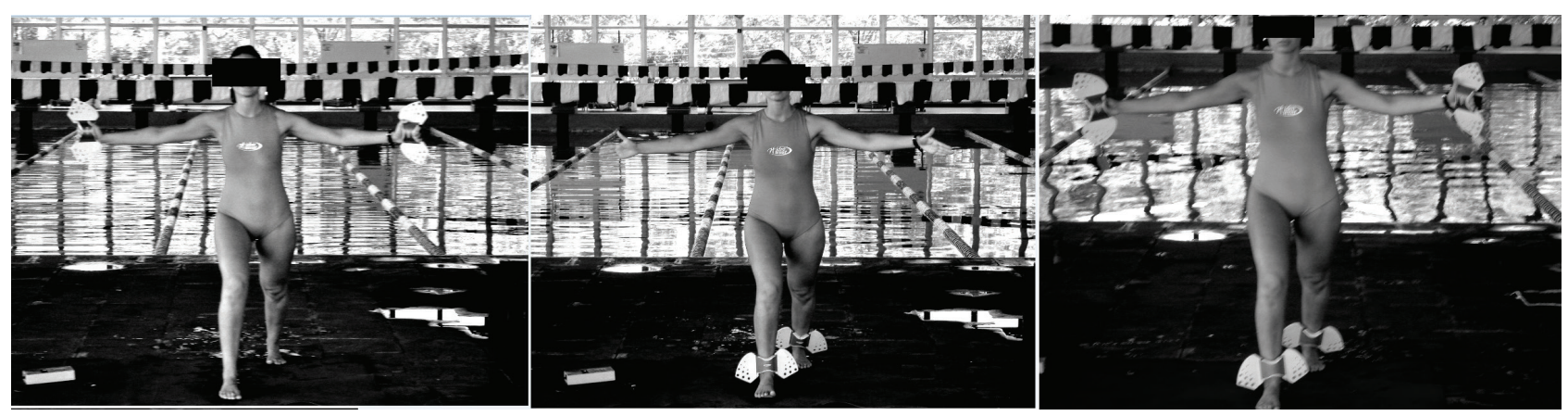

Figura 1 - Exercício deslize frontal combinado com a flexão e extensão horizontal de ombros nas diferentes situações: (A) uso de equipamento nos membros superiores (MS), (B) uso de equipamento nos membros inferiores (MI) e (C) uso de equipamento nos membros superiores e inferiores (MS/MI).

O equipamento nos membros inferiores foi cuidadosamente localizado $3 \mathrm{~cm}$ acima do maléolo medial, com as abas posicionadas no plano frontal proporcionando uma resistência aos movimentos anteroposteriores durante o exercício deslize frontal. Nos membros superiores os indivíduos seguravam o equipamento na palma das mãos com as abas posicionadas com intuito de proporcionar uma resistência aos movimentos contralaterais durante o exercício de flexão e extensão horizontal de ombros.

Em todas as situações, foi verificada a $\mathrm{FC}$, o consumo de oxigênio $\left(\mathrm{VO}_{2}\right)$, a ventilação $\left(V_{E}\right)$ e o IEP geral. Para a verificação da FC foi utilizado um frequencímetro (modelo S610, POLAR, Kajaani, Finlândia). A fim de medir o $\mathrm{VO}_{2}$ e a $\mathrm{V}_{\mathrm{E}}$ foi utilizado um analisador de gases portátil (modelo KB1-C, AEROSPORT, Ann Arbor, EUA), o qual foi calibrado de acordo com instruções do fabricante. Os valores dessas variáveis foram obtidos a cada 20 s. A verificação do IEP geral, que pode ser definida como um esforço percebido que está intimamente relacionado com as respostas do sistema musculoesquelético, cardiovascular e pulmonar, foi realizado através da escala de Borg ${ }^{27}$ ao final de cada situação de exercício. Tal escala (reproduzida em um banner, 60x90 cm), estava fixada fora da piscina, posicionada imediatamente a frente dos sujeitos.

Os dados cardiopulmonares foram analisados através da média dos valores coletados entre o terceiro e o quarto minuto da execução do exercício em cada situação, visto que nesse período as variáveis cardiopulmonares se encontram no seu estado estável, de acordo com estudo prévio realizado com exercícios de hidroginástica ${ }^{29}$. Além disso, foi calculado o percentual do $\mathrm{VO}_{2 \text { máx }}\left(\% \mathrm{VO}_{2 \text { máx }}\right)$ e da $\mathrm{FC}_{\text {máx }}$ $\left(\% \mathrm{FC}_{\text {máx }}\right)$ nas diferentes situações através dos valores de referência de $\mathrm{VO}_{2 \operatorname{máx}} \mathrm{e}$ $\mathrm{FC}_{\text {máx }}$ obtidos no teste máximo. Para relacionar as variáveis cardiopulmonares e o IEP foi utilizado o teste de correlação de Spearman, com um nível de significância de 5\%. O pacote estatístico utilizado foi o SPSS versão 18.0.

\section{RESULTADOS}

Das 11 mulheres que realizaram o protocolo experimental, quatro iniciaram o 
mesmo pela realização do exercício deslize frontal combinado com a flexão e extensão horizontal de ombros com o uso de equipamento em MS, quatro iniciaram com o uso de equipamento em MI e três iniciaram com o uso de equipamento em MS/MI. Observou-se correlações significativas (rho=0,60-0,88; $\mathrm{p}<0,05$ ) entre o IEP e as variáveis cardiopulmonares $\left(\mathrm{FC}, \% \mathrm{FC}_{\text {maxx }}, \mathrm{VO}_{2}, \% \mathrm{VO}_{2 \text { máx }}\right.$ e $\mathrm{V}_{\mathrm{E}}$ ) na situação MI (Figura 2). Todavia, nas demais situações (MS e MS/MI) não houve correlações significativas entre esses parâmetros, com exceção da associação entre o IEP e a $V_{E}$ na situação $\mathrm{MS} / \mathrm{MI}$, a qual apresentou um rho=0,71 significativo $(\mathrm{p}=0,015)$. Esses resultados podem ser visualizados na tabela 1 .

IEP $\times$ FC

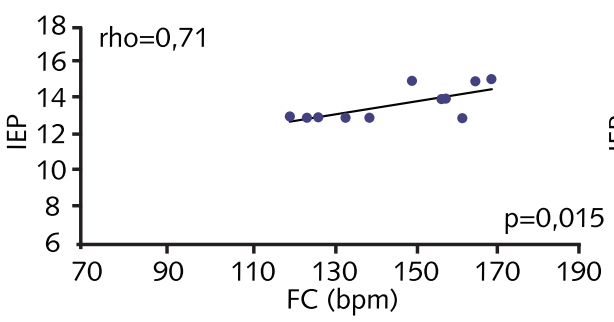

$\mathrm{IEP} \times \mathrm{VO}_{2}$

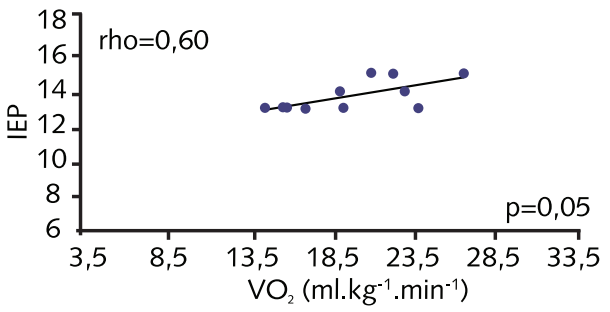

IEP $\times \% \mathrm{FC}_{\text {max }}$

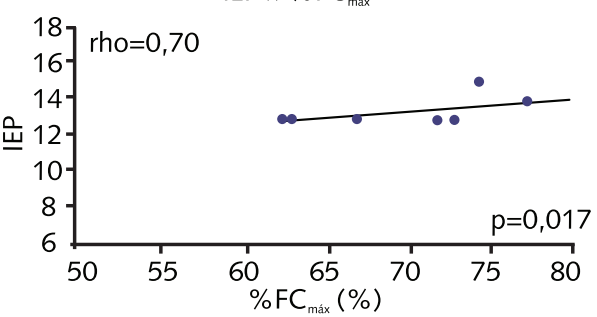

$\mathrm{IEP} \times \% \mathrm{VO}_{2 \max }$

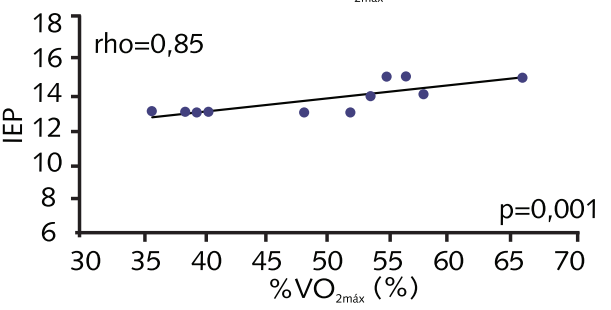

IEP $\times V$

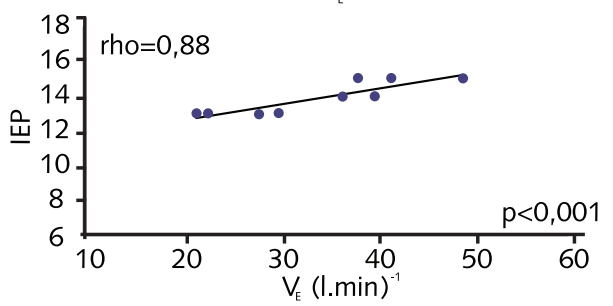

Figura 2 - Correlação entre o índice de esforço percebido (IEP) e frequência cardíaca (FC) (A), IEP e percentual da frequência cardíaca máxima $\left(\% \mathrm{FC}_{\text {máx }}\right)(\mathrm{B})$, IEP e consumo de oxigênio $\left(\mathrm{VO}_{2}\right)(\mathrm{C})$, IEP e percentual do consumo de oxigênio máximo $\left(\% \mathrm{VO}_{2 \operatorname{máx}}\right)(\mathrm{D})$ e IEP e ventilação $\left(\mathrm{V}_{\mathrm{E}}\right)$. p: nível de significância; rho: coeficiente de correlação.

Tabela 1 - Relação entre índice de esforço percebido (IEP) e frequência cardíaca (FC), percentual da frequência cardíaca máxima ( $\% \mathrm{FC}_{\text {máx }}$ ), consumo de oxigênio $\left(\mathrm{VO}_{2}\right)$, percentual do consumo de oxigênio máximo $\left(\% \mathrm{VO}_{2 \operatorname{máx}}\right)$ e ventilação $\left(\mathrm{V}_{\mathrm{E}}\right)$ nas situações de uso de equipamento nos membros superiores e nos membros superiores e inferiores.

\begin{tabular}{lcccc} 
& \multicolumn{2}{c}{$\begin{array}{c}\text { Equipamento nos membros } \\
\text { superiores }\end{array}$} & \multicolumn{2}{c}{$\begin{array}{c}\text { Equipamento nos membros } \\
\text { superiores e inferiores }\end{array}$} \\
\hline $\mathrm{IEP} \times \mathrm{FC}$ & rho & $\mathrm{p}$ & rho & $\mathrm{p}$ \\
\hline $\mathrm{IEP} \times \% \mathrm{FC}_{\text {máx }}$ & $-0,049$ & 0,877 & $-0,033$ & 0,923 \\
\hline $\mathrm{IEP} \times \mathrm{VO}_{2}$ & $-0,051$ & 0,882 & $-0,245$ & 0,468 \\
\hline $\mathrm{IEP} \times \% \mathrm{VO}_{\text {máx }}$ & $-0,083$ & 0,808 & $-0,080$ & 0,815 \\
\hline $\mathrm{IEP} \times \mathrm{V}_{\mathrm{E}}$ & 0,042 & 0,903 & 0,433 & 0,183 \\
\hline
\end{tabular}

Nota - rho: coeficiente de correlação de Spearman. ${ }^{*} p<0,05$ 


\section{DISCUSSÃO}

Os principais achados do presente estudo foram as correlações significativas observadas entre o IEP e as variáveis cardiopulmonares na situação de realização do exercício de hidroginástica com equipamento resistido apenas nos membros inferiores. Por outro lado, esse comportamento entre o IEP e parâmetros cardiopulmonares não foi observado nas demais situações.

A correlação positiva e significativa encontrada no presente estudo na situação MI corrobora estudos da literatura que também investigaram exercícios aquáticos. Svedenhag \& Seger ${ }^{19}$ demonstraram que o $\mathrm{VO}_{2}$ e o IEP aumentam linearmente com $o$ incremento da intensidade durante a corrida em piscina funda. Da mesma forma, Shono et al..$^{20}$ observaram correlação significativa de 0,99 entre a FC e o IEP durante a caminhada em esteira subaquática. Com exercícios de hidroginástica, destacam-se dois estudos da literatura. Alberton et al. ${ }^{22}$ investigaram três exercícios de hidroginástica (corrida estacionária, chute frontal e deslize lateral) e observaram correlações significativas entre o IEP e o $\mathrm{VO}_{2}(\mathrm{r}=0,65 ; \mathrm{p}<0,001)$ e entre o IEP e o \% $\mathrm{VO}_{2 \text { máx }}(\mathrm{r}=0,68$; $\mathrm{p}<0,001)$. Em outro estudo ${ }^{21}$, analisando apenas o exercício corrida estacionária na hidroginástica, os autores reportaram correlações significativas entre o IEP e diferentes parâmetros fisiológicos, tal como a $\mathrm{FC}(\mathrm{r}=0,65 ; \mathrm{p}<0,001)$, o \% $\mathrm{FC}_{\text {máx }}(\mathrm{r}=0,65$; $\mathrm{p}<0,001)$, o $\mathrm{VO}_{2}(\mathrm{r}=0,60 ; \mathrm{p}=0,001), o \% \mathrm{VO}_{2 \text { máx }}(\mathrm{r}=0,71 ; \mathrm{p}<0,001)$ e a $\mathrm{V}_{\mathrm{E}}(\mathrm{r}=0,77$; $\mathrm{p}<0,001)$. No presente estudo também foi observado na situação MI correlações que variaram de moderada a forte ${ }^{30}$ ( $\left.\mathrm{rho}=0,60-0,88\right)$, ressaltando que as correlações que apresentaram valores maiores de rho também foram as associações do IEP com o $\% \mathrm{VO}_{2 \text { max }}(\mathrm{rho}=0,85)$ e com a $\mathrm{V}_{\mathrm{E}}(\mathrm{rho}=0,88)$, assim como no estudo previamente citado. Além disso, Alberton et al..$^{21}$ também reportaram que esses dois parâmetros são os que melhor explicaram o IEP geral através de uma análise de regressão múltipla (79\%). Tal comportamento pode ser explicado pelo fato de que o IEP apresenta uma relação linear com parâmetros cardiopulmonares quando existe uma grande massa muscular de membros inferiores envolvida no exercício. Isso ocorre devido ao fato dessa situação de exercício aquático impactar de maneira significativa na capacidade do sistema sensorial e proprioceptivo perceber a intensidade de esforço de maneira similar à demanda dos sistemas cardiovascular, musculoesquelético e pulmonar.

Em contrapartida, a falta de correlação entre o IEP e as variáveis cardiopulmonares nas situações MS e MS/MI, provavelmente possa ser explicada pelo fato de que a percepção do exercício executado com equipamento nos membros superiores foi influenciada pela percepção de esforço localizada (MS e MS/MI). Provavelmente isso possa ser explicado pelo fato da área projetada do equipamento (Aquafins ${ }^{\circledR}$ utilizado tanto em MS quanto em MI) representar relativamente uma maior sobrecarga neuromuscular para os membros superiores em comparação aos membros inferiores. Dessa forma, especula-se que o uso de equipamento em MS tenha influenciado o IEP geral, especialmente por uma alteração mais proeminente do sistema muscular (IEP local) em comparação a mesma observada nos sistemas pulmonar e cardiovascular (IEP respiratório). Esses resultados corroboram parcialmente o estudo de Raffaelli et al. ${ }^{31}$ que, ao analisar cinco exercícios de hidroginástica (corrida estacionária, deslize lateral, deslize frontal, chute frontal e chute lateral), observou uma correlação fraca $(\mathrm{r}=0,44)$, porém significativa $(p<0,001)$ entre o IEP geral e o $\mathrm{VO}_{2}$. Possivelmente essa fraca relação deve-se ao fato de que a análise foi realizada agrupando diferentes exercícios de hidroginástica, os quais apresentavam distintos grupos e tamanhos musculares envolvidos (flexores e extensores de quadris e joelhos vs. adutores e abdutores de quadris). 
A partir desses resultados, sugere-se que para exercícios envolvendo uma grande massa muscular de membros inferiores com o uso de equipamento resistido, o IEP geral pode ser efetivo para predizer a intensidade do exercício em jovens experientes em atividades aquáticas. Todavia, o uso do mesmo equipamento nos membros superiores parece alterar o IEP geral, possivelmente devido ao IEP local de membros superiores. Logo, o IEP geral parece não ser adequado para predizer a intensidade de esforço quando se utiliza equipamento nos membros superiores.

A principal limitação do presente estudo está relacionada ao fato de terem sido investigadas apenas mulheres jovens. Logo, cautela é necessária para a aplicabilidade de tais resultados em outras populações, tais como homens e sujeitos idosos. Além disso, em relação à abordagem metodológica, possivelmente a mensuração das variáveis cardiopulmonares no repouso nas três situações de exercício (MS, MI e MS/MI) garantiria efetivamente que os sujeitos partiram do mesmo estado metabólico antes de cada situação. Cabe destacar que nesse estudo foi apenas analisado um exercício e um tipo de equipamento, logo, mais estudos são necessários para aprofundar o conhecimento acerca de tais respostas em outros exercícios e equipamentos amplamente utilizados nas aulas de hidroginástica.

\section{CONCLUSÃO}

Os resultados do presente estudo indicam que o IEP está associado com a frequência cardíaca, com o consumo de oxigênio e com a ventilação, e reflete a intensidade do exercício de hidroginástica quando realizado com equipamento resistido nos membros inferiores em mulheres jovens. Todavia, as situações de uso de equipamentos apenas nos membros superiores ou combinando o uso em membros superiores e inferiores durante exercício de hidroginástica não apresentaram relações significativas entre o IEP e os parâmetros cardiopulmonares.

\section{Agradecimentos}

Agradecemos à CAPES e ao CNPq pelo apoio na execução desse projeto.

\section{REFERÊNCIAS}

1. Carvalho VO, Bocchi EA, Guimarães GV. The Borg Scale as an important tool of selfmonitoring and self-regulation of exercise prescription in heart failure patients during hydrotherapy: A randomized blinded controlled trial. Circ J. 2009; 73(10):1871-6.

2. Jones LM, Meredith-Jones K, Legge M. The effect of water-based exercise on glucose and insulin response in overweight women: A pilot study. J Womens Health. 2009; 18(10):1653-9.

3. Bento PCB, Pereira G, Ugrinowitsch C, Rodacki ALF. The effects of a water-based exercise program on strength and functionality of older adults. J Aging Phys Act. 2012; 20:469-83.

4. Srámek P, Simecková M, Janski L, Savlíková J, Vybíral S. Human Physiological responses to immersion into water of different temperatures. Eur J Appl Physiol. 2000; 81(5):436-42.

5. Alberton CL, Finatto P, Pinto SS, Antunes AH, Cadore EL, Kruel LFM. Comparação das respostas cardiorrespiratórias de repouso entre os meio terrestre e aquático. Rev Bras Ativ Fis e Saúde. 2013; 18(3):387-95.

6. Azevedo, LB, Lambert, MI, Zogaib, OS, and Barros Neto, TL. Maximal and submaximal physiological responses to adaptation to deep water running. J Sports Sci. 2010; 28(4):407-14

7. Alberton CL, Tartaruga MP, Pinto SS, Cadore EL, Antunes AH, Finatto P, et al. Vertical ground reaction force during water exercises performed at different intensities. Int J Sports Med. 2013; 34(10):881-7.

8. Batterham SI, Heywood S, Keatinge JL. Systematic review and meta-analysis comparing land and aquatic exercises for people with hip or knee arthritis on function, mobility and other health outcomes. BMC Musculoskelet Disord. 2011; 12:123.

9. Alexander R. Mechanics and energetic of animal locomotion. In: Alexander R, Goldspink G (eds). Swimming. London: Chapman \& Hall; 1977. p. 222-48 
10. Alberton CL, Olkoski MM, Becker ME, Pinto SS, Kruel LFM. Cardiorespiratory responses of postmenopausal women to different water exercises. Int J Aquatic Res Educ. 2007; 1(4):363-72.

11. Alberton CL, Cadore EL, Pinto SS, Tartaruga MP, Silva EM, Kruel LFM. Cardiorespiratory, neuromuscular and kinematic responses to stationary running performed in water and on dry land. Eur J Appl Physiol. 2011; 111(6):1157-66.

12. Pinto SS, Cadore EL,Alberton CL, Silva EM, Kanitz AC, Tartaruga MP, et al. Cardiorespiratory and neuromuscular responses during water aerobics exercise performed with and without equipment. Int J Sports Med. 2011; 32(12):916-23.

13. Pinto SS, Cadore EL, Alberton CL, Zaffari P, Bagatini NC, Baroni BM, et al. Effects of intrasession exercise sequence during water-based concurrent training. Int J Sports Med. 2013; doi: 10.1055/s-0033-1345129.

14. Tsourlou T, Benik A, Dipla K, Zafeiridis A, Spiros K. The Effects of a twenty-four-week aquatic training program on muscular strength performance in healthy elderly women. J Strength Cond Res. 2006; 20(4):811-8.

15. Kruel LFM, Posser MS, Alberton CL, Pinto SS, Oliveira AS. Comparison of energy expenditure between continuous and interval water aerobic routines Int J Aquatic Res Educ. 2009; 3:186-96.

16. Robertson RJ, Noble BJ. Perception of physical exertion: Methods, mediators, and applications. Exerc Sport Sci Rev. 1997; 25:407-52.

17. Graef FI, Kruel LFM. Frequência cardíaca e percepção subjetiva do esforço no meio aquático: diferenças em relação ao meio terrestre e aplicações na prescrição do exercício - uma revisão. Rev Bras Med Esporte. 2006;12(4):221-8.

18. Swank AM, Steinel L, Moore A. Strategies for effectively using ratings of perceived exertion. Strength \& Conditioning Journal. 2003; 25(4):23-5.

19. Svedenhag J, Seger J. Running on land and in water: comparative exercise physiology. Med Sci Sports Exerc. 1992; 24(10):1155-60.

20. Shono T, Fujishima K, Hotta N, Ogaki T, Ueda T, Otoki K. Physiological responses and RPE during underwater treadmill walking in women of middle and advanced age. J Physiol Anthropol Appl Human Sci. 2000; 19(4):195-200.

21. Alberton CL, Antunes AH, Beilke DD, Pinto SS, Kanitz AC, Tartaruga MP, et al. Maximal and ventilatory threshold of oxygen uptake and rating of perceived exertion responses to water aerobic exercises. J Strength Cond Res. 2013; 27(7):1897-903.

22. Alberton CL, Rothmann CRC, Pinto SS, Coertjens M, Kruel LFM. Consumo de oxigênio e índice de esforço percebido em diferentes ritmos de execução na hidroginástica. Motriz. 2012; 18(3):423-31.

23. Pinto SS, Alberton CL, Becker ME, Olkoski MM, Kruel LFM. Respostas cardiorrespiratórias em exercícios de hidroginástica executados com e sem o uso de equipamento resistivo. Rev Port Ciênc Desp. 2006; 6(3):336-41.

24. Costa G, Afonso S, Bragada JA, Reis VM, Barbosa TM. Estudo comparativo das adaptações fisiológicas agudas durante a execução de três variantes de m exercício básico de hidroginástica. Rev Bras Cineantropom Desempenho Humano. 2008; 10(4):323-29.

25. Pinto SS, Alberton CL, Figueiredo PAP, Tiggemann CL, Kruel LFM. Respostas de frequência cardíaca, consumo e oxigênio e sensação subjetiva ao esforço em um exercício de hidroginástica executado por mulheres em diferentes situações com e sem o equipamento Aquafins ${ }^{\circledR}$. Rev Bras Med Esp. 2008; 14(4):357-61.

26. Howley ET, Basset Jr DR, Welch HG. Criteria for maximal oxygen uptake: review and commentary. Med Sci Sports Exerc. 1995; 27(9):1292-301.

27. Borg G. Psychophysical scaling with applications in physical work and the perception of exertion. Scand J Work Environ Health. 1990; 16(1):55-58.

28. Cooke CB. Metabolic rate and energy balance. In: Eston R, Reilly T (eds) Kinanthropometry and exercise physiology laboratory manual. London: E \& FN Spon; 1996. p. 175-95.

29. Moraes EZC, Kruel LFM, Sampedro RMF, Lopes LFD. Metodologia de medida de esforço para exercícios de hidroginástica em diferentes profundidades de água. Revista Kinesis. 2002; 27:43-64.

30. Callegari-Jacques SM. Bioestatística: princípios e aplicações. Porto Alegre: Artmed, 2003.

31. Raffaelli C, Galvani C, Lanza M, Zamparo P. Different methods for monitoring intensity during water-based aerobic exercises. Eur J Appl Physiol. 2012; 112(1):125-34.

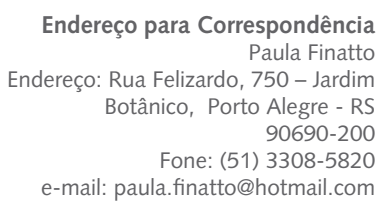

